\title{
Single-session Curative Embolization of Unruptured Cerebellar Arteriovenous Malformation with Precipitating Hydrophobic Injectable Liquid
}

\author{
Giancarlo Saal-Zapata1, Dante Valer ${ }^{1}$ Rodolfo Rodriguez ${ }^{1}$
}

Address for correspondence Giancarlo Saal-Zapata, MD, Department of Neurosurgery, Endovascular Neurosurgery Service, Hospital Nacional Guillermo Almenara Irigoyen - EsSalud, La Victoria, Grau Avenue 800, La Victoria, Lima, Perú (e-mail: gian_carlo1987@hotmail.com,gsaal1987@gmail.com).

\begin{abstract}
Keywords

- cerebellar arteriovenous malformation

- precipitating hydrophobic injectable liquid embolization

Microsurgery is the treatment of choice for cerebellar arteriovenous malformations (AVMs). Nevertheless, endovascular therapy aims to be an effective treatment since the introduction of new embolic agents. Cerebellar AVMs represent a subgroup of vascular lesions with a high rate of hemorrhagic presentation compared with their cerebral counterpart. Moreover, they have a higher incidence of flow-related aneurysms when diagnosed. With this in mind, prompt treatment must be performed to avoid rebleeding with devastating consequences. Herein, we present the case of an unruptured cerebellar AVM treated with precipitating hydrophobic injectable liquid (PHIL) with complete obliteration.
\end{abstract}

\section{Introduction}

Posterior fossa arteriovenous malformations (AVM) represent 10 to $15 \%$ of all AVMs, whereas cerebellar AVMs represent around the $75 \%$ of all posterior fossa AVMs. ${ }^{1,2}$ The most frequent clinical presentation is hemorrhage in around 60 to $80 \%$ of the cases, which may present as parenchymal, intraventricular, or subarachnoid.

The gold standard treatment of this lesions is microsurgery, with high rates of total obliteration, but moderate morbidity and mortality. ${ }^{3}$ Nevertheless, other options are available such as endovascular embolization and radiosurgery.

Little is known about the curative results of endovascular embolization of posterior fossa AVMs because it is mainly used as an adjuvant therapy prior to microsurgery or radiosurgery. ${ }^{4}$ A lack of clinical studies and case reports exist regarding the endovascular management of posterior fossa AVMs. Moreover, new embolic agents and endovascular devices are increasingly used to treat this pathology more frequently.

To the best of our knowledge, this is the first report of an immediate and midterm follow-up of complete obliteration of a posterior fossa AVM following embolization with precipitating hydrophobic injectable liquid (PHIL; Microvention, Tustin, CA, United States).

\section{Case Report}

In a previously healthy 39-year-old woman, with a 5-year history of headache that improves with analgesics, MRI is performed, revealing a left petrous unruptured cerebellar AVM.

The patient visits the emergency department of our hospital because of headache and vomiting, a brain CT scan is performed ruling out bleeding. She is hospitalized in the emergency department. Digital subtraction angiography (DSA) is performed, revealing a cerebellar AVM with feeders arising from the left posterior cerebral artery (PCA), left anterior superior cerebellar artery (ASCA), and left anterior inferior cerebellar artery (AICA), with venous drainage to the straight and transverse sinus and an associated large venous aneurysm. The AVM dimensions were $20 \mathrm{~mm} \times 18 \mathrm{~mm}$. We decided to perform embolization through the left AICA.
DOI https://doi.org/

$10.1055 / \mathrm{s}-0039-3403457$

ISSN 2277-954X.
License terms

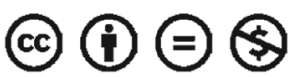


Endovascular procedure: The right femoral artery was approached by Seldinger's technique.

A guide catheter Boston $6 \mathrm{~F}$ was navigated to the right vertebral artery; then, a Headway Duo microcatheter (Microvention), Hybrid 007 microwire (Balt Extrusion, Montmorency, France), and liquid embolic agent PHIL (Microvention) were used. The microcatheter was navigated over the microwire into the left AICA, and then a superselective angiogram was performed showing the AVM nidus and the venous drainage. Once in the appropriate position, embolization was performed with $4 \mathrm{~mL}$ of liquid embolic agent PHIL, achieving good penetration to the nidus. A control DSA showed absence of the nidus with little reflux to the left AICA, and there was also absence of the venous aneurysm and the venous drainage.

After the embolization, the patient developed sixth cranial nerve paresis, hearing loss, left dysdiadochokinesia, nausea and vomiting, but no motor deficit. A brain CT scan was performed and showed no bleeding. She was discharged home 7 days after the procedure. A 9-month follow-up DSA demonstrated total obliteration of the AVM nidus and disappearance of the previous impairments.

\section{Discussion}

Current treatment of AVMs includes microsurgery, endovascular therapy, radiosurgery, and a combination of these modalities, depending on multiple features. It is well-established that microsurgery is the treatment of choice for cerebellar AVMs, and embolization is mainly indicated prior to surgery or prior to radiosurgery as an adjuvant treatment to reduce the blood flow and size of the nidus.

Compared with supratentorial AVMs, these lesions carry a higher risk of morbidity and mortality: the high-rate of rupture with compromise of vital structures and hydrocephalus must be a cause of concern. ${ }^{5}$

The standard classification system for AVMs is the Spetzler--Martin classification, which predicts the complication rate when surgery is performed, but not when endovascular approach is chosen. The Spetzler-Martin classification is the most widely used in all types of AVMs, ${ }^{6}$ especially supratentorial AVMs, but is not suitable for posterior fossa lesions. This can be explained because the only eloquent structures in the cerebellum are the deep nuclei, and the deep venous drainage is not a good indicator of AVM depth.

Currently, there is no standard classification for cerebellar AVMs.

From the morphological and anatomical perspective, Rodriguez-Hernández et al classified the cerebellar AVMs into five types: suboccipital, vermian, tonsillar, tentorial, and petrosal. ${ }^{7}$ This can be correlated with angiographic studies and helps in preoperative planification.

From the endovascular counterpart, the Buffalo Score is a newly developed system that predicts complications if
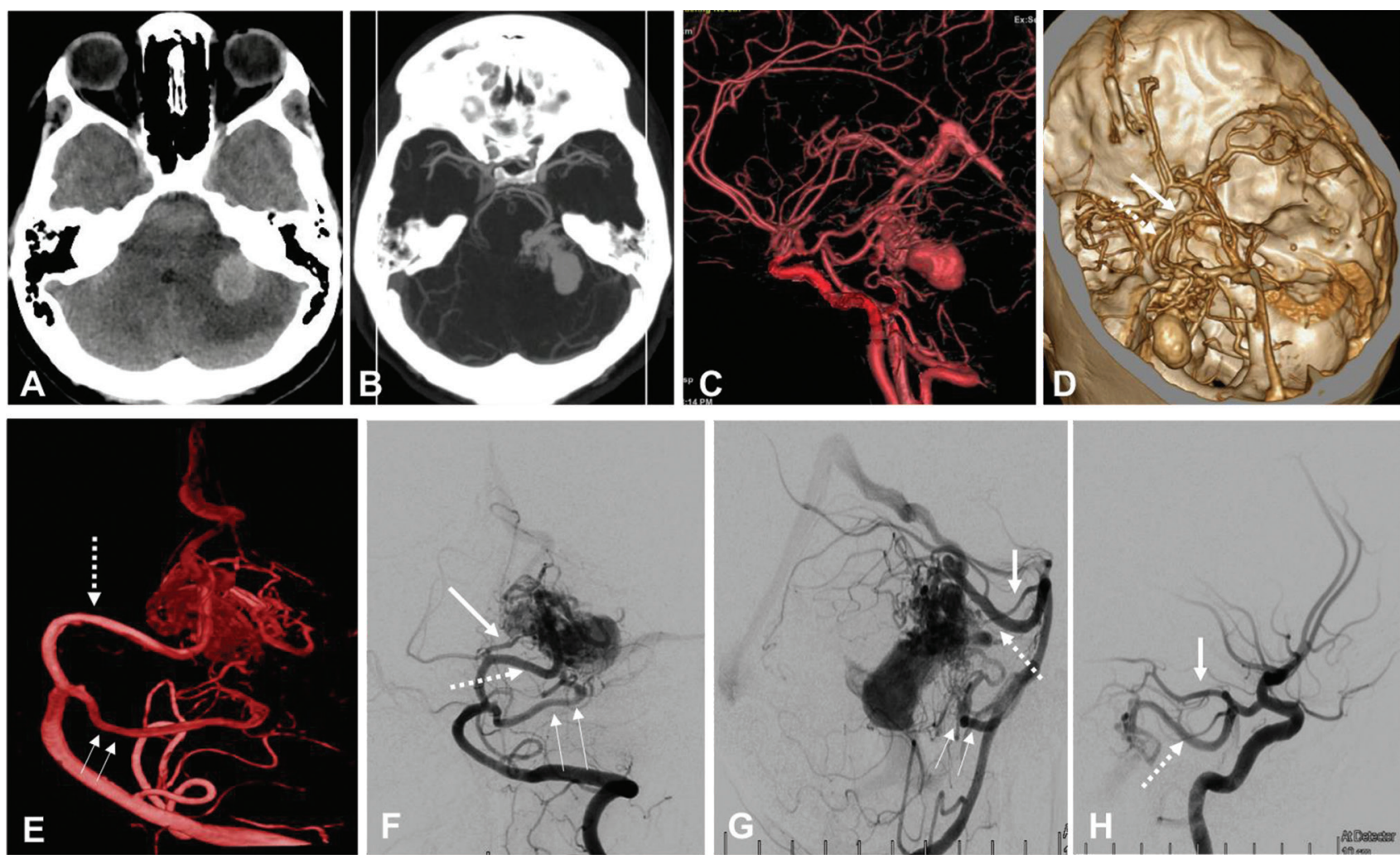

Fig. 1 Brain CT scan revealing vasogenic edema with an aneurysm (A). CTA confirms the presence of a nidus with an associated aneurysm (B). CTA with three-dimensional reconstruction shows the presence of a cerebellar AVM with a venous aneurysm (C); feeders from the posterior cerebral artery (PCA) and ASCA can be seen (D). DSA of the left vertebral artery with three-dimensional reconstruction shows feeders from the left ASCA and the AICA (double arrow) (E). DSA shows the three feeders (PCA, ASCA, and AICA) with an associated large venous aneurysm and drainage to the straight sinus $(\mathbf{F}, \mathbf{G}, \mathbf{H})$. PCA = arrow, ASCA = dotted arrow, AICA = double arrow. AICA, anterior inferior cerebellar artery; ASCA, anterior superior cerebellar artery; AVM, arteriovenous malformation; PCA, posterior cerebral artery. 
endovascular treatment is selected. ${ }^{8}$ Unfortunately, this score does not include posterior fossa AVMs.

In selected cases, endovascular therapy can cure these complex lesions. New embolic agents such as PHIL were developed for this purpose. This novel product is a nonadhesive dimethyl sulfoxide (DMSO)-based liquid embolic agent with iodine for radiopacity. ${ }^{9}$ This product has some advantages over Onyx (Medtronic Neurovascular, Irvine, CA, USA). ${ }^{10}$

Posterior fossa AVMs should be treated promptly because of the high-rate of rupture compared with supratentorial AVMs. ${ }^{5}$

The association with aneurysms occurs in $20 \%$ of all AVMs and ranges from 5 to $20 \%$. $^{11,12}$

If the aneurysms are located in the infratentorial space, the risk of rupture rises to $60 \%{ }^{11}$

Possible complications during treatment are rupture, infarctions, and hydrocephalus. Moreover, these lesions can cause hydrocephalus due to mechanical obstruction in the ventricular system. ${ }^{13}$
Robert et al treated 69 patients with posterior fossa AVMs, achieving an obliteration rate of $72.5 \%$ with subsequent microsurgery or radiosurgery in 15 patients. ${ }^{14}$

Feng et al treated a total of 63 patients with posterior fossa AVM, of which 20 received endovascular treatment. Of these 20 patients, only two patients achieved complete obliteration. ${ }^{4}$

At our institution, we treat all AVMs with endovascular therapy. In this case, we chose the embolic agent PHIL because of its properties to penetrate the nidus.

The patient of this report did not fortunately develop any vascular complications. The initial complaints were due to the mass effect of the embolic agent and its relation with surrounding neurovascular structures.

We do not believe that endovascular therapy is an adjunct treatment for cerebellar AVMs. As many papers describe, it is mainly used prior to microsurgery and radiosurgery to reduce the size. We think that in well-selected cases, the embolization of the AVM could achieve complete obliteration, as in our
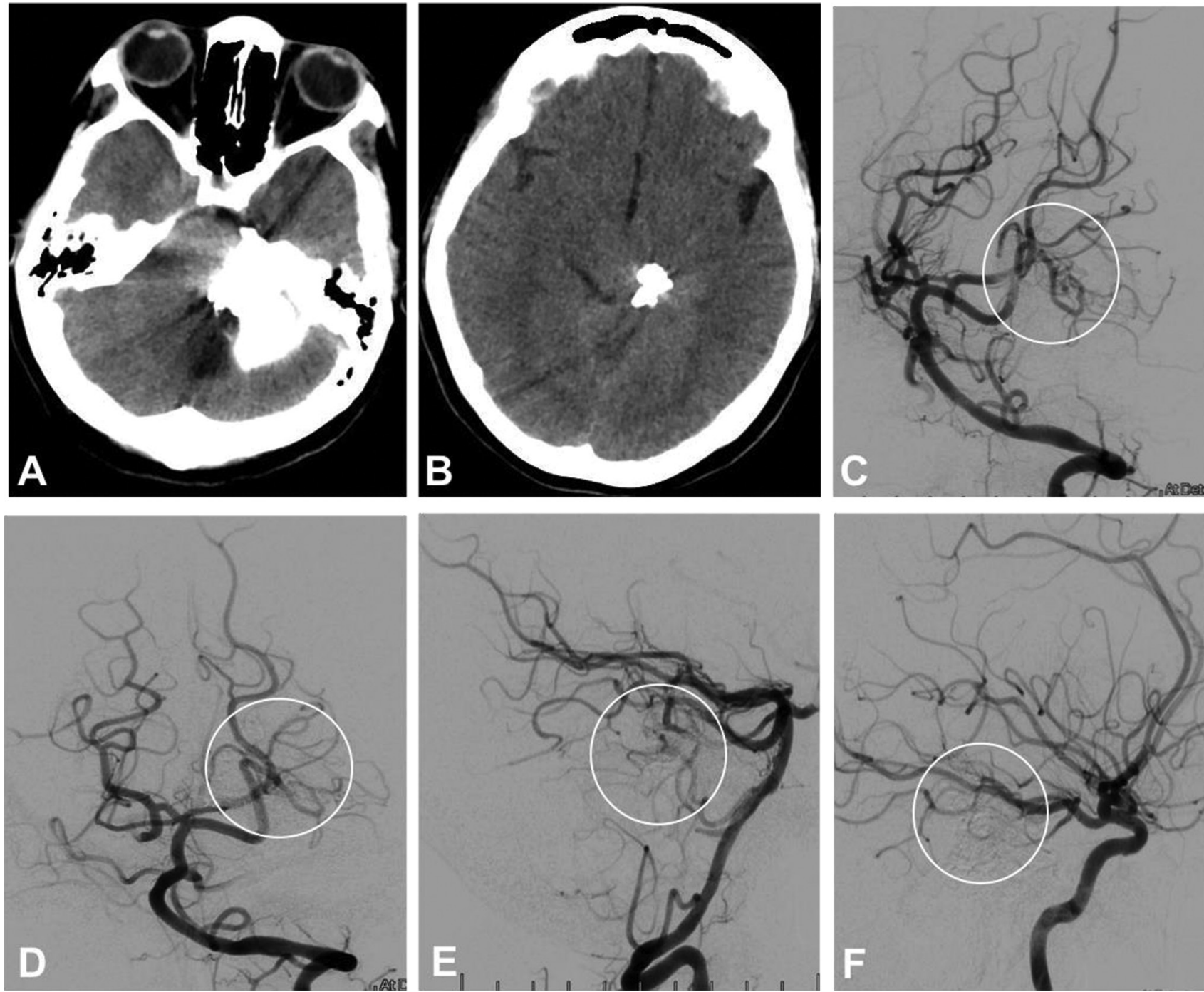

Fig. 2 Brain CT postembolization showing embolic agent artifact and no bleeding (A, B). DSA of the left vertebral artery revealing complete obliteration of the nidus without venous drainage and venous aneurysm. The procedure was performed through the left AICA (C, D, E). DSA of the left ICA showing absence of the nidus (F). Abbreviations: AICA, anterior inferior cerebellar artery; ICA, internal carotid artery; DSA, digital subtraction angiography. 
case. It will depend on the feeding vessels, angioarchitecture of the nidus, and location within the posterior fossa.

\section{Conclusions}

Microsurgery is the treatment of choice for cerebellar AVMs; nevertheless, endovascular treatment is an option in selected cases with curative results.

Currently, endovascular neurosurgeons are provided with a wide armamentarium of endovascular devices, such as liquid embolic agents that allow penetration to the AVM nidus with complete obliteration and achievement of definitive cure.

\section{Conflict of Interest}

None declared.

\section{References}

1 Almeida JP, Medina R, Tamargo RJ. Management of posterior fossa arteriovenous malformations. Surg Neurol Int 2015;6:31

2 Arnaout OM, Gross BA, Eddleman CS, Bendok BR, Getch CC, Batjer HH. Posterior fossa arteriovenous malformations. Neurosurg Focus 2009;26(5):E12

3 Torné R, Rodríguez-Hernández A, Arikan F, et al. Posterior fossa arteriovenous malformations: Significance of higher incidence of bleeding and hydrocephalus. Clin Neurol Neurosurg 2015;134:37-43

4 Lai LF, Chen JX, Zheng K, et al. Posterior fossa brain arteriovenous malformations: Clinical features and outcomes of endovascular embolization, adjuvant microsurgery and radiosurgery. Clin Neuroradiol 2018;28(1):17-24

5 Dinc N, Platz J, Tritt S, et al. Posterior fossa AVMs: increased risk of bleeding and worse outcome compared to supratentorial AVMs. J Clin Neurosci 2018;53:171-176
6 Spetzler RF, Martin NA. A proposed grading system for arteriovenous malformations. J Neurosurg 1986;65(4):476-483

7 Rodríguez-Hernández A, Kim H, Pourmohamad T, Young WL, Lawton MT; University of California, San Francisco Arteriovenous Malformation Study Project. Cerebellar arteriovenous malformations: anatomic subtypes, surgical results, and increased predictive accuracy of the supplementary grading system. Neurosurgery 2012;71(6):1111-1124

8 Dumont TM, Kan P, Snyder KV, Hopkins LN, Siddiqui AH, Levy EI. A proposed grading system for endovascular treatment of cerebral arteriovenous malformations: Buffalo score. Surg Neurol Int 2015;6(1):3

9 Vollherbst DF, Sommer CM, Ulfert C, Pfaff J, Bendszus M, Möhlenbruch MA. Liquid embolic agents for endovascular embolization: evaluation of an established (Onyx) and a novel (PHIL) embolic agent in an in vitro AVM model. Am J Neuroradiol 2017;38(7):1377-1382

10 Vollherbst DF, Otto R, von Deimling A, et al. Evaluation of a novel liquid embolic agent (precipitating hydrophobic injectable liquid (PHIL)) in an animal endovascular embolization model. J Neurointerv Surg 2018;10(3):268-274

11 CagnazzoF,BrinjikjiW,Lanzino G. Arterial aneurysms associated with arteriovenous malformations of the brain: classification, incidence, risk of hemorrhage, and treatment-a systematic review. Acta Neurochir (Wien) 2016;158(11):2095-2104

12 Redekop G, TerBrugge K, Montanera W, Willinsky R. Arterial aneurysms associated with cerebral arteriovenous malformations: classification, incidence, and risk of hemorrhage. J Neurosurg 1998;89(4):539-546

13 Diren F, Sencer S, Hakan T. Case report of an obstructive hydrocephalus caused by an unruptured mesencephalic arteriovenous malformation in a boy and a review of literature. Open Neuroimaging J 2018;12:10-15

14 Robert T, Blanc R, Ciccio G, et al. Endovascular treatment of posterior fossa arteriovenous malformations. J Clin Neurosci 2016;25:65-68 